\section{$\underset{\substack{\text { hommes } \\ \text { \& migrations }}}{ }$}

\section{Hommes \& migrations}

Revue française de référence sur les dynamiques

migratoires

1285 | 2010

L'appel du pied

\title{
La migration des footballeurs africains en France
}

Le cas des Ivoiriens (1957-2010)

\section{Claude Boli}

\section{(2) OpenEdition}

12 Journals

Édition électronique

URL : http://journals.openedition.org/hommesmigrations/1178

DOI : 10.4000/hommesmigrations. 1178

ISSN : 2262-3353

Éditeur

Musée national de l'histoire de l'immigration

Édition imprimée

Date de publication : 1 mai 2010

Pagination : 58-64

ISSN : 1142-852X

\section{Référence électronique}

Claude Boli, « La migration des footballeurs africains en France», Hommes \& migrations [En ligne], 1285 | 2010, mis en ligne le 29 mai 2013, consulté le 03 mai 2019. URL : http://

journals.openedition.org/hommesmigrations/1178; DOI : 10.4000/hommesmigrations.1178

Tous droits réservés 


\section{La migration des footballeurs africains en France \\ Le cas des lvoiriens (1957-2010)}

Par Claude Boli, historien et sociologue, responsable des départements Recherche et Collection au Musée national du sport

Depuis les années cinquante, dans le contingent de joueurs venus d'Afrique noire, la présence des footballeurs ivoiriens s'est maintenue et représente, un demi-siècle plus tard, l'une des plus importantes dans les championnats français de ligue 1 et 2. De Jean Tokpa "I'Alésien"

à Bakary Koné l'un des joyaux des "académiciens", en passant par l'incontournable Didier Drogba, et les Bleus Basile Boli ou Djibril Cissé, le terrain du football est l'expression d'une longue histoire et des relations singulières entre la Côte d'Ivoire et la France. 
"En AOF ; Football et chasse à l'éléphant. Quinze mille kilomètres en huit jours, quatre victoires, succès complet, impression profonde', voici résumé en style télégraphique la tournée de nos deux équipes amateurs en Afrique occidentale française. C'est la première fois qu'une sélection de la métropole venait dans ces régions. Le football, malgré les difficultés du climat, y est devenu populaire ${ }^{(1)} . .$. Importé par les colons français, le football s'est développé en Côte d'Ivoire durant les années trente et quarante. La colonisation par le jeu a été promue par les aventuriers de l'expansion coloniale: militaires, ecclésiastiques, commerçants, enseignants, voyageurs. Restreint à l'élite blanche, le football s'est progressivement étendu à la population locale, d'abord aux cadres autochtones désireux d'embrasser le sport des maîtres, et ensuite aux jeunes garçons de conditions très modestes. Au milieu des années trente se créent trois clubs pionniers. Sous contrôle de colons français se fonde la Planification industrielle Christian (PIC), l'Union sportive des fonctionnaires d'Abidjan (USFA) qui est une fusion entre Français et Ivoiriens, et l'Union sportive des indigènes (USI) entièrement formée par les fonctionnaires ivoiriens. En 1947 sont fondés les principaux clubs ivoiriens, l'ASEC d'Abidjan et l'Africa Sport d'Abidjan. C'est de ces deux équipes que proviennent les premiers Ivoiriens du championnat de France. Les villes des alentours de Sète, Alès, Montpellier constituent les principales zones d'arrivée de Jean Tokpa, Benjami Akouaté, Sékou Touré.

\section{La filière sudiste, 1957-1972}

En août 1957, la Une de France football fait état de "l'escadron noir du FC Sète". Parmi les six joueurs présents, figurent les Ivoiriens Anoh, Yapi, Akouaté et Zokoua. Au milieu des années cinquante, des difficultés économiques poussent les clubs hexagonaux à un recrutement de joueurs non onéreux. À l'été 1956, sous les conseils de Guy Fabre, Sétois émigré en Côte d'Ivoire et entraîneur d'une équipe corporative, Louis Michel, le président du club de Sète, décide de faire venir Ignace Wognin et Gaston Zokoua, deux des meilleurs éléments de l'équipe "corpo ${ }^{(2)}$ ". Le président risquait peu puisque le prix de leur transfert était juste un billet de voyage par bateau Abidjan-Sète. "L'opération Côte d'Ivoire" comme elle est appelée dans l'hebdomadaire France football apporte une grande satisfaction. Les deux joueurs s'adaptent parfaitement aux réalités du football professionnel, et participent au sauvetage du club en seconde division.

De cette première vague d'Ivoiriens, celui qui marqua les esprits de l'opinion publique est certainement Jean Tokpa. C'est le "chef de file" des footballeurs ivoiriens, 
comme l'écrit Max Urbini de France football. Prévu à Alès, il est "détourné" par un dirigeant de Montpellier à l'été 1955. À Montpellier, puis à Sète, il s'illustre par ses talents de buteur. Lors des saisons $1957-58$ et 1958-59, avec Alès en première division, il inscrit 31 buts en 65 rencontres. Très vite, il devient l'un des joueurs emblématiques de l'impact des footballeurs venus d'Afrique noire.

"Perle noire", "sorcier", "le Brésilien", "le magnifique", les qualificatifs explosent. Une campagne médiatique est menée pour qu'il intègre l'équipe de France en Coupe du monde 1958, étant considéré français en tant que membre de la Communauté française. Gabriel Hanot, l'une des voix du football français, l'élit meilleur attaquant à la fin de la saison 1958-59. En juillet 1959 il est transformé en vedette quand il signe dans la grande équipe du Racing Club de France pour la somme de 26 millions de francs (transfert record à l'époque). Le footballeur n'intéresse pas que les journaux. Sa vie privée est divulguée dans la presse, notamment son mariage et les avis de son épouse sur sa carrière. À l'instar de vedettes de l'époque, une paire de chaussures de football porte son nom. Après cinq saisons au Racing, Jean Tokpa met fin à sa carrière et rentre en Côte d'Ivoire pour devenir entraîneur de l'équipe nationale.

\section{Le moment Pokou, 1973-1983}

Après les réussites de Tokpa et d'autres talents qui ont bâti leur réputation dans le sud de la France, plusieurs recruteurs hexagonaux vont s'intéresser de plus près au championnat ivoirien lancé en 1960. Les excellentes prestations de l'équipe nationale et des clubs dans les compétitions africaines augmentent l'intérêt d'une attention particulière au football ivoirien. En 1966, le stade d'Abidjan remporte la seconde édition de la toute récente Coupe d'Afrique des Clubs Champions. Deux années plus tard, lors de la CAN (Coupe d'Afrique des nations) organisée en Éthiopie, les Ivoiriens réalisent un excellent parcours en terminant à la troisième place. Un jeune joueur ivoirien est la révélation du tournoi : il s'agit de Laurent Pokou, formé par l'entraîneur français Paul Gévaudan, entraîneur des Éléphants de Côte d'Ivoire. La presse va s'intéresser à celui qui termine en tête des meilleurs buteurs avec six réalisations. Le nom de Pokou sort progressivement du cercle des initiés du football ivoirien pour devenir un nom connu en France. En 1970, dans une volonté de promouvoir l'universalité du football, France football lance le Ballon d'or africain qui couronne le meilleur joueur du continent. Laurent Pokou termine à la seconde place, devancé par le Malien Salif Keita. Jean Philipe Rethacker, le 
"monsieur Afrique" de l'hebdomadaire écrit : "Il est évident que ce gaillard d'une classe exceptionnelle a reçu, depuis deux ans, bon nombre de propositions alléchantes de la part de clubs pros français. Ainsi Mario Zatelli et Marseille en 1968, Nantes ensuite, Robert Domergue et Monaco enfin, à Khartoum, lui offrirent de signer un contrat pro. Mais Pokou ne se laissa pas convaincre, préférant rester à Abidjan où il bénéficie de grands avantages matériels et, surtout, d'une gloire, d'une popularité assez extraordinaires ${ }^{(3)}$."

En décembre 1973, après plusieurs années d'hésitations ${ }^{(4)}$ et d'offres refusées de clubs français, Laurent Pokou accepte de venir en France, au Stade Rennais plus précisément, afin de poursuivre sa carrière de footballeur. En trois saisons à Rennes, il impressionna journalistes, supporters, dirigeants et joueurs. Peu de temps après ses débuts, il fait l'unanimité auprès de la presse footballistique qui le place parmi les meilleurs attaquants mondiaux. Ses exploits sont soigneusement relatés dans la presse locale (Ouest France) et nationale. Le "Pelé africain" est le surnom qu'il reçoit de

\section{En 1970, dans une volonté de promouvoir l'universalité du football, France football lance le Ballon d'or africain qui couronne le meilleur joueur du continent.} France football. Un entraîneur le comparait au fameux Ben Barek, le fabuleux joueur marocain des années quarante. La saison 1975-76 est celle où il démontre ses qualités de finisseur hors pair en marquant dix-sept buts en douze rencontres. La carrière de Pokou prend fin brutalement en 1979. Lors d'une rencontre de Coupe de France, le joueur est accusé d'avoir porté volontairement un coup à l'arbitre. Le joueur clame son innocence mais il est suspendu deux ans puis six mois. Pokou crie au racisme ${ }^{(5)}$. Des blessures récurrentes l'obligent à écourter sa carrière française.

\section{Désir de France : Monaco un club "ivoirien", 1984-1992}

Les années quatre-vingt marquent le début de l'exil des meilleurs joueurs du championnat ivoirien. Un désir de France se fait de plus en plus remarquer dans l'esprit de footballeurs peu connus comme très connus. Les transferts de Youssouf Fofana en 1984, d'Abdoulaye Traoré en 1988 constituent de véritables moments cruciaux.

En 1984, Youssouf Fofana, alors âgé de 18 ans, signe à l'AS Cannes entraîné par Jean-Marc Guillou. Le transfert du joueur est fortement débattu. Il faut l'aval du président Houphouët-Boigny pour permettre le départ de celui qui fit ses débuts à 
l'ASEC d'Abidjan à l'âge de 16 ans. Après quelques mois à Cannes, il rejoint l'AS Monaco où il est pris sous la coupe d'Arsène Wenger. Avec Youssouf Fofana s'entament diverses relations avec la Côte d'Ivoire qui aboutissent à un "partenariat" entre le club monégasque et l' ASEC d'Abidjan. Des joueurs évoluant en Côte d'Ivoire sont suivis par les dirigeants monégasques. Dans le cadre de cette relation privilégiée, plusieurs joueurs ivoiriens sont appelés à faire des essais. Le club monégasque devient le "destinataire" potentiel des meilleurs éléments de l'équipe de la capitale ivoirienne. Une aide matérielle (ballons, chaussures) permet aux deux clubs de tirer profit de cette situation.

En 1988, Abdoulaye Traoré le meilleur joueur ivoirien de la fin des années quatrevingt décide de venir en France. Il signe à Toulon en 1988 puis poursuit une courte carrière à Metz. Son parcours français se solde par un échec. Plusieurs joueurs vont également choisir de migrer en France, en temps de crise du football ivoirien, notamment à cause du recul des investissements des présidents.

\section{Ivoire en Bleus et Bleus en ivoire, 1993-2004}

En 1993, quand l'Olympique de Marseille remporte le premier trophée d'un club français dans une compétition européenne, l'originaire de Côte d'Ivoire Basile Boli est le héros de la finale. Naturalisé français, il est le premier joueur d'origine ivoirienne à porter le maillot bleu de l'équipe de France, et aussi l'auteur du but qui permet aux Marseillais de soulever la prestigieuse Coupe d'Europe des Clubs Champions. L'enfant d'Adjamé (quartier de la capitale ivoirienne) arrive en France pour poursuivre sa scolarité en 1982 à l'âge de 15 ans. Sous l'influence de son frère Roger, il prend une licence au CA Romainville, club de la banlieue est parisienne. C'est lors d'une rencontre de championnat de France cadet qu'il est repéré par les clubs d'Auxerre, Sochaux, Nantes et de Paris Saint-Germain. Il signe finalement à l'AJ Auxerre où il effectue neuf saisons au sein de la défense. Cependant, la carrière de Boli est fortement liée à la Coupe d'Europe avec l'Olympique de Marseille. Grâce à ce titre, il va connaître une notoriété tant auprès des médias sportifs que non sportifs (premier footballeur invité dans l'émission 7 sur 7). Élevé comme un symbole de "la France qui gagne" et surtout de la France de la diversité, il est approché par le gouvernement socialiste pour jouer un rôle de trait d'union entre la France et la Côte d'Ivoire lors de certains voyages diplomatiques.

Djibril Cissé et Abou Diaby, tous deux nés respectivement à Arles et à Paris, sont d'autres joueurs originaires de Côte d'Ivoire qui brillent en Bleus. 
Depuis les années deux mille, l'équipe de Côte d'Ivoire est composée de joueurs nés et formés en France. Choix de raison et de cceur se mêlent dans les décisions des joueurs qui optent pour la sélection de Côte d'Ivoire. Emerse Faé (avant lui Boris Diecket) né à Nantes, formé au FC Nantes, champion du monde des moins de 17 ans en 2001 avec l'équipe de France, opte pour la sélection ivoirienne "pour avoir une chance de disputer une Coupe du monde". 


\section{Une présence affirmée, 2005-2010}

À l'été 2005, la rencontre amicale entre la France et la Côte d'Ivoire à Montpellier permet de découvrir six pensionnaires de l'académie de football créée en 1994 par le Français Jean-Marc Guillou. Les journalistes créent autour d'eux le mythe d'une génération exceptionnelle. La Côte d'Ivoire devient tout à coup le pays qui possède un réservoir de joueurs prodigieux. Parmi ces joueurs, il y a Bakari Koné. Le petit "ailier de poche" est né en 1981. Il a été repéré dans le quartier de Williamsville par Jean-Marc Guillou. C'est en 1994 qu'il rentre à l'académie. En 2002, il est prêté au club Al Ittihad (Qatar). Une année plus tard il se pose à Lorient pour deux ans, puis rejoint l'OGC Nice pour deux autres années, avant de signer à l'Olympique de Marseille.

Il est pratiquement impossible de parler des footballeurs ivoiriens aujourd'hui sans évoquer Didier Drogba. En mai 2010, le mensuel américain Time le désigne parmi les 100 personnalités les plus influentes de la planète. En tant que footballeur, l'image de Drogba est liée à celle de l'Olympique de Marseille et dans une moindre mesure au club où il débuta, au Mans, à Guingamp. Hors du stade, Drogba est investi dans des causes humanitaires.

Les réussites des Drogba, Koné ne doivent pas faire oublier des centaines de jeunes joueurs qui, au prix d'énormes sacrifices des parents, ont voulu tenter l'expérience étrangère par l'entremise d'agents. Des rêves de joueurs brisés par des agents peu scrupuleux qui rappellent les difficultés de devenir footballeur professionnel.

\section{Conclusion}

Les Ivoiriens constituent une composante importante du paysage footballistique français. Ils représentent l'un des principaux groupes parmi les 67 joueurs africains de la ligue 1 lors de la saison 2009-2010. Grâce à l'exceptionnelle réussite de Didier Drogba, ils sont l'attraction de nombreux recruteurs étrangers. Les Ivoiriens se distinguent autant par leur présence dans les clubs hexagonaux que par leur place dans l'équipe nationale française. La Coupe du monde 2010 offre l'occasion de voir les prestations des Ivoiriens de France qui évoluent avec les Éléphants et aussi des Français de Côte d'Ivoire qui brillent avec les Coqs gaulois.

\section{Notes}

1. France football, 30 mai $1950, \mathrm{n}^{\circ} 219$.

2. France football, 20 août $1957, \mathrm{n}^{\circ} 596$.

3. France football, 5 janvier $1971, \mathrm{n}^{\circ} 1292$.

4. France football, 3 mars $1970, \mathrm{n}^{\circ} 1248$.

5. France football, 23 janvier $1979, n^{\circ} 1711$. 